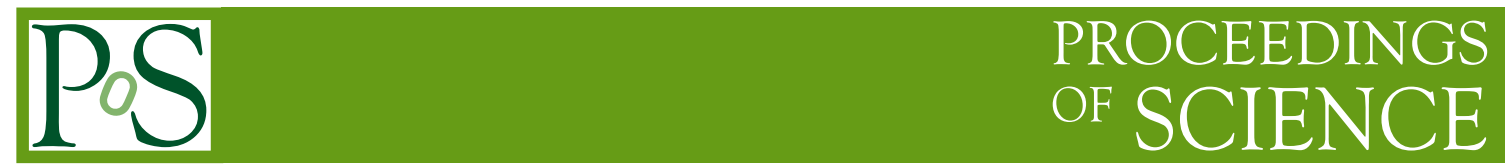

\title{
Radiative decays at LHCb
}

\section{Ivan Belyaev* for LHCb Collaboration}

Nikhef, National Institute for Subatmic Physics, Amsterdam, Netherlands Intitute for Theoretical and Experimental Physics, Moscow, Russia

E-mail: Ivan.Belyaeveitep.ru

We discuss the potential of LHCb to discover New Physics in radiative decays of beauty hadrons. In particular the measurement of the photon polarization in radiative decays $\mathrm{B}_{\mathrm{s}}^{0} \rightarrow \phi \gamma$ is discussed, where the expected uncertainty of the photon polarization parameter $\sin (2 \psi)$ is 0.22 for $2 \mathrm{fb}^{-1}$.

12th International Conference on B-Physics at Hadron Machines - BEAUTY 2009 September 07 - 112009

Heidelberg, Germany

* Speaker. 


\section{Introduction}

The principal aim of the LHC is to discover new physical interactions and to probe their nature. There are two main approaches to New Physics (NP) searches: direct and indirect. Within the direct approach, one could expect new particles to be produced and observed as real paticles at energy frontier machines (e.g. LHC). But, indirect searches are also a powerful approach, since new particles can give additional contributions to loop or box diagrams through which the transitions occur: their existence could then be probed by measuring the decay rate and kinematic distributions. Rare decays in the beauty sector encompass a wide range of processes and constitute a sensitive tool in the search for NP as well as in precision measurements of the Standard Model (SM) parameters, e.g. the Cabibbo-Kobayashi-Maskawa (CKM) matrix elements. The focus of this paper is on processes with final states containing photons in addition to daughter hadron(s). The examples considered include the electromagnetic or electroweak penguin decays $B \rightarrow K^{*} \gamma$ and $B_{s} \rightarrow \phi \gamma$. These rare decays correspond to diagrams with internal loops leading to effective flavor-changing neutral current (FCNC) transitions. The presence of new virtual particles (e.g. supersymmetric ones) may manifest itself in altering the decay rate, $C P$ asymmetry and other observable quantities. Exciting new perspectives are emerging in these studies owing to the very large statistics that will be collected by the LHCb experiment, which will enable us to enter a new realm of high precision studies of rare $B$ decays.

\subsection{Experimental Strategies}

The goal of many of the measurements considered in this review is to measure the amplitude ratio $\frac{\mathscr{A}\left(\mathrm{B} \rightarrow \mathrm{f} \gamma_{\mathrm{R}}\right)}{\mathscr{A}\left(\mathrm{B} \rightarrow \mathrm{f} \gamma_{\mathrm{L}}\right)}$ where $\mathrm{f}$ represents some exclusive final hadronic state. There is no experimental possibility of measuring photon polarization directly, but there are several indirect strategies. The first one is the study of the angular distributions of the $f$ decay products $[1,2]$. In this way one is able to measure only the square of the amplitude ratio. Indeed, using the definition of the photon polarization parameter $\lambda_{\gamma}=\frac{\left|\mathscr{A}_{R}\right|^{2}-\left|\mathscr{A}_{L}\right|^{2}}{\left|\mathscr{\ell}_{R}\right|^{2}+\left|\mathscr{A}_{L}\right|^{2}}$, one has

$$
\frac{\mathrm{d} \Gamma(\mathrm{B} \rightarrow \mathrm{f} \gamma)}{\mathrm{d} \Omega} \propto\left(\left|\mathscr{A}_{\mathrm{R}}\right|^{2}+\left|\mathscr{A}_{\mathrm{L}}\right|^{2}\right)+\lambda_{\gamma}\left(\left|\mathscr{A}_{\mathrm{R}}\right|^{2}-\left|\mathscr{A}_{\mathrm{L}}\right|^{2}\right),
$$

where $\mathscr{A}_{\mathrm{L}, \mathrm{R}}$ corresponds to the amplitudes for left and right handed photons. It is worth noticing that the amplitudes $\mathscr{A}_{\mathrm{R}, \mathrm{L}}$ do not interfere since the polarization of the photon in the final state can (at least in principle) be measured independently. By studying the angular distribution one can extract $\lambda_{\gamma}$ from (1.1), in other words the method makes use of angular correlations among the decay products $^{1}$ in $\mathrm{B} \rightarrow\left[\Phi \rightarrow \mathrm{P}_{1} \mathrm{P}_{2} \mathrm{P}_{3}\right] \gamma$, where $\mathrm{P}_{\mathrm{i}}$ is either a pion or a kaon. This technique was originally suggested in $[1,2]$ and used for the decay $\mathrm{B} \rightarrow K \pi \pi \gamma$ with the sum over intermediate hadron resonances. The radiative decay mode $\mathrm{B} \rightarrow\left[\varphi \rightarrow \mathrm{K}^{+} \mathrm{K}^{-}\right] \mathrm{K} \gamma$ is considered in [3]. This mode is rather distinctive with many desirable features from the experimental point of view: the final state is a photon plus only charged mesons (for charged B mesons), the fact that $\varphi$ is narrow reduces the effects of intermediate resonances interference etc. However the actual situation is rather involved. The possibility of measuring $\lambda_{\gamma}$ in this way depends on the precise nature of the interfering ampli-

\footnotetext{
${ }^{1}$ Note that there must be at least three particles in the final state.
} 
tudes. The arrangement of these amplitudes may be unfavorable and the asymmetry may escape detection [3].

Alternatively, one can study baryon decays such as $\Lambda_{\mathrm{b}} \rightarrow \Lambda^{0} \gamma \rightarrow \mathrm{p} \pi \gamma\left(\right.$ or $\left.\Lambda_{\mathrm{b}} \rightarrow \Lambda^{*} \gamma \rightarrow \mathrm{pK} \gamma\right)$ and measure the photon polarization via the forward-backward asymmetry of the proton with respect to the $\Lambda_{b}$ in the $\Lambda^{0}$ rest frame for polarized $\Lambda_{b}$, (see $[4,5,6]$ for details and references therein).

Aside from the experimental difficulties, the main problem of these two methods is the absence of interference between the amplitudes for left- and right-handed photon emission, since they correspond to different and distinguishable (at least in principle) final states. Correspondingly they are sensitive only to the square of the amplitude ratio in the form of $\lambda_{\gamma}$. In order to access the ratio of the raw amplitudes themselves it is necessary to use other approaches. There are two ways in which this may be done. One makes use of the fact that some photons convert in the detector material into electron-positron pairs. It can be shown that for these processes the distribution in the angle $\chi$ between the $\mathrm{e}^{+} \mathrm{e}^{-}$plane and the plane defined by the final state hadrons (e.g. K $\pi$ resulting from $\mathrm{K}^{*}$ decay) should be isotropic for purely circular polarization, while deviations from this isotropy are driven by the same parameter $\mathscr{A}_{\mathrm{R}} / \mathscr{A}_{\mathrm{L}}$, indicating the presence of right-handed photons $[7,8,9,10]$. In principle however, multiple scattering makes it difficult to identify the decay plane for low invariant mass $\mathrm{e}^{+} \mathrm{e}^{-}$-pairs. This is not the case for pair creation from virtual photons where one can select pair masses above $30 \mathrm{MeV} / \mathrm{c}^{2}$ without losing too much rate. However in this case other diagrams contribute with longitudinal virtual photons. LHCb prospects for this measurement are discussed elsewhere [11].

Another way to obtain sestitivity to $\mathscr{A}_{\mathrm{R}} / \mathscr{A}_{\mathrm{L}}$ is to study the time evolution of $\mathrm{B}_{(\mathrm{s})}^{0} \rightarrow \mathrm{f}^{C P} \gamma$ decays, where $\mathrm{f}^{C P}$ is some $C P$-eigenstate. In this case the time-dependent decay rates can be conventionally parameterized as follows:

$$
\begin{aligned}
& \Gamma_{B_{(s)}^{0} \rightarrow \mathrm{f} C \gamma}(t)=|A|^{2} e^{-\Gamma_{(s)} t}\left(\cosh \frac{\Delta \Gamma_{(s)} t}{2}-\mathscr{A}^{\Delta} \sinh \frac{\Delta \Gamma_{(s)} t}{2}+C \cos \Delta m_{(s)} t-S \sin \Delta m_{(s)} t\right) \\
& \Gamma_{\bar{B}_{(s)}^{0} \rightarrow \mathrm{f}^{C P} \gamma}(t)=|A|^{2} e^{-\Gamma_{(s)} t}\left(\cosh \frac{\Delta \Gamma_{(s)} t}{2}-\mathscr{A}^{\Delta} \sinh \frac{\Delta \Gamma_{(s)} t}{2}+C \cos \Delta m_{(s)} t+S \sin \Delta m_{(s)} t\right)
\end{aligned}
$$

Within the SM one has [12]:

$$
C \approx 0, \quad S \approx \sin 2 \psi \sin \varphi_{(\mathrm{s})}, \quad \mathscr{A}^{\Delta} \approx \sin 2 \psi \cos \varphi_{(\mathrm{s})},
$$

where $\psi$ is defined as

$$
\tan \psi \equiv\left|\frac{\mathscr{A}\left(\overline{\mathrm{B}}_{(\mathrm{s})} \rightarrow \mathrm{f}^{C P} \gamma_{\mathrm{R}}\right)}{\mathscr{A}\left(\overline{\mathrm{B}}_{(\mathrm{s})} \rightarrow \mathrm{f}^{C P} \gamma_{\mathrm{L}}\right)}\right|
$$

and related to the fraction of "wrongly"-polarized photons ${ }^{2}$; and $\varphi_{(\mathrm{s})}$ is the sum of $\mathrm{B}_{(\mathrm{s})}^{0}$ mixing phase and $C P$-odd weak phases for right $\mathscr{A}_{\mathrm{R}}$ and left $\mathscr{A}_{\mathrm{L}}$ amplitudes. From (1.4) and (1.5) one can see that the measurement of $\mathscr{A}^{\Delta}$ and $S$ directly determines the "wrongly"-polarized photon fraction [12].

\footnotetext{
${ }^{2}$ Note that the parameter $\lambda_{\gamma}$ can be expressed as $\lambda_{\gamma}=\cos 2 \psi$.
} 
For the $\mathrm{B}^{0}$ system the parameter $\Delta \Gamma$ is totally negligible, and thus the terms proportional to $\mathscr{A}^{\Delta}$ vanish:

$$
\begin{aligned}
& \Gamma_{B^{0} \rightarrow \mathrm{f} C P}(t) \approx|A|^{2} e^{-\Gamma t}\left(1-S \sin \Delta m_{(s)} t\right), \\
& \Gamma_{\bar{B}^{0} \rightarrow \mathrm{f}^{C P} \gamma}(t) \approx|A|^{2} e^{-\Gamma t}\left(1+S \sin \Delta m_{(s)} t\right) .
\end{aligned}
$$

Furthermore in the $\mathrm{SM}$ one expects $\sin \varphi=\sin \left(2 \beta-\phi_{\mathrm{p}}\right) \approx \sin 2 \beta$, where $\phi_{\mathrm{p}}$ is a $C P$-odd weak penguin phase. Therefore one gets:

$$
S_{\mathrm{B}^{0}}=\sin 2 \psi \sin 2 \beta
$$

On the contrary for the $\mathrm{B}_{\mathrm{s}}^{0}$ system the parameter $\Delta \Gamma_{\mathrm{s}}$ is not negligible, providing a non-zero sensitivity to $\mathscr{A}^{\Delta}$. In the $\operatorname{SM} \varphi_{\mathrm{s}}$ is expected to be small, $\sin \varphi_{\mathrm{s}}=\sin \left(2 \beta_{\mathrm{s}}-\phi_{\mathrm{p}}\right) \approx 0$, thus the term with $S$ vanishes:

$$
\Gamma_{B_{s}^{0} \rightarrow \Phi^{C P} \gamma}(t) \approx|A|^{2} e^{-\Gamma_{s} t}\left(\cosh \frac{\Delta \Gamma_{s} t}{2}-\mathscr{A}^{\Delta} \sinh \frac{\Delta \Gamma_{s} t}{2}\right)
$$

and finally one gets:

$$
\mathscr{A}_{\mathrm{B}_{\mathrm{s}}{ }^{0}}^{\Delta} \approx \sin 2 \psi
$$

thus opening the possibility for a direct measurement of the photon polarization parameter $\sin 2 \psi$ [13]. It is worth stressing here that for vanishing $S$ and $C$, both $\mathrm{B}_{\mathrm{s}}^{0}$ and $\overline{\mathrm{B}}_{\mathrm{s}}^{0}$ exhibit the same decay time evolution and therefore no flavour tagging is required for the extraction of $\sin 2 \psi$.

\subsection{Selected studies of radiative decays at $\mathrm{LHCb}$}

A list of interesting measurements with radiative decays of b-hadrons includes:

1. the measurement of the photon polarization and $C P$-violation parameters $C$ and $S$ in the decay $\mathrm{B}_{\mathrm{s}}^{0} \rightarrow \phi \gamma[14,15,16,17]$,

2. the measurement of the photon polarization in the decays of polarized beauty baryons $\Lambda_{b} \rightarrow$ $\Lambda^{0} \gamma$ and $\Lambda_{\mathrm{b}} \rightarrow\left(\Lambda^{*} \rightarrow \mathrm{pK}^{-}\right) \gamma[18,19,20]$,

3. the measurement of the photon polarization in the decay $\mathrm{B}^{+} \rightarrow \phi \mathrm{K}^{+} \gamma$,

4. the measurement of direct $C P$-violation in the decay $\mathrm{B}^{0} \rightarrow \mathrm{K}^{* 0} \gamma$,

5. the precise measurement of the ratio of branching fractions for $\mathrm{B}_{\mathrm{s}}^{0} \rightarrow \phi \gamma$ and $\mathrm{B}^{0} \rightarrow \mathrm{K}^{* 0} \gamma$ decays.

Some of these items have been intensively studied in $\operatorname{LHCb}[14,15,16,17,18,19,20]$, while for other topics, e.g. for the decay $\mathrm{B}^{+} \rightarrow \phi \mathrm{K}^{+} \gamma$, only initial studies of the event selection have been performed.

The event selection criteria described in detail elsewhere $[15,16,17,18,19]$, have been chosen to maximize the ratio $\frac{S}{\sqrt{S+B}}$. The reconstruction efficiency for decay $\mathrm{B}_{\mathrm{s}}^{0} \rightarrow \phi \gamma \varepsilon_{\mathrm{rec}}$, the selection efficiency for reconstructed events $\varepsilon_{\mathrm{sel} / \mathrm{rec}}$, the L0-trigger efficiency for selected events $\varepsilon_{\mathrm{L} 0 / \mathrm{sel}}$ and the total efficiency $\varepsilon_{\text {tot }}$ are summarized in Table 1. 
Table 1: The reconstruction efficiency $\varepsilon_{\text {rec }}$, selection efficiency for reconstructed events $\varepsilon_{\mathrm{sel} / \mathrm{rec}}$ and the L0-trigger efficiency for selected $\mathrm{B}_{\mathrm{s}}^{0} \rightarrow \phi \gamma$ events $\varepsilon_{\mathrm{L} 0 / \text { sel }}[15]$.

\begin{tabular}{lc}
\hline \hline Efficiency & $\mathrm{B}_{\mathrm{s}}^{0} \rightarrow \phi \gamma$ \\
\hline$\varepsilon_{\mathrm{rec}}$ & $1.9 \%$ \\
$\varepsilon_{\mathrm{sel} / \mathrm{rec}}$ & $11.7 \%$ \\
$\varepsilon_{\mathrm{L} 0 / \mathrm{sel}}$ & $44.1 \%$ \\
\hline$\varepsilon_{\mathrm{tot}}$ & $0.10 \%$ \\
\hline \hline
\end{tabular}

Table 2 gives the expected nominal event yields for a sample corresponding to $2 \mathrm{fb}^{-1}$ of integrated luminosity and the estimates of the background to signal ratio $\left(B_{\mathrm{b} \overline{\mathrm{b}}} / S\right)$ for the above mentioned radiative $B$ decays. Here the background level has been estimated form a large sample of inclusive $b \bar{b}$-simulation. Such events are expected to be the dominant source of contamination.

Table 2: The expected yields per $2 \mathrm{fb}^{-1}$ of integrated luminosity and background to signal ratios, $B_{\mathrm{b} \overline{\mathrm{b}}} / S$, for radiative decays of beauty hadrons. The backgrounds only include backgrounds from b $\bar{b}$-events. Upper limits are quoted at $90 \% \mathrm{CL}$.

\begin{tabular}{llcc}
\hline \hline Decay Mode & & Yield & $B_{\mathrm{b} \overline{\mathrm{b}}} / S$ \\
\hline \hline $\mathrm{B}^{0} \rightarrow \mathrm{K}^{* 0} \gamma$ & {$[15]$} & $7 \times 10^{4}$ & $0.6-0.7$ \\
$\mathrm{~B}_{\mathrm{s}}^{0} \rightarrow \phi \gamma$ & {$[15]$} & $1.1 \times 10^{4}$ & $<0.6-0.9$ \\
$\Lambda_{\mathrm{b}} \rightarrow \Lambda^{0} \gamma$ & {$[18]$} & 750 & $<42$ \\
$\Lambda_{\mathrm{b}} \rightarrow \Lambda^{0}(1670) \gamma$ & {$[18]$} & $2.5 \times 10^{3}$ & $<18$ \\
$\Lambda_{\mathrm{b}} \rightarrow \Lambda^{0}(1690) \gamma$ & {$[19]$} & $2.5 \times 10^{3}$ & $<18$ \\
$\Lambda_{\mathrm{b}} \rightarrow \Lambda^{0}(1520) \gamma$ & {$[19]$} & $4.3 \times 10^{3}$ & $<9$ \\
$\mathrm{~B}^{+} \rightarrow \phi \mathrm{K}^{+} \gamma$ & & $\sim 7 \times 10^{3}$ & $<2-4$ \\
\hline \hline
\end{tabular}

Assuming the nominal detector performance we expect to observe the $\mathrm{B}_{\mathrm{s}}^{0} \rightarrow \phi \gamma$ signal mass peak with an effective resolution of $98 \mathrm{MeV} / c^{2}[15,16]$. The width is dominated by the resolution of the electromagneric calorimeter $[14,15,21]$. The expected statistics for $\mathrm{B}^{0} \rightarrow \mathrm{K}^{* 0} \gamma$ and $\mathrm{B}_{\mathrm{s}}^{0} \rightarrow \phi \gamma$ decays for the accumulated luminosity of $2 \mathrm{fb}^{-1}$ (see Table 2) will allow us to perform a precise measurement of the actual signal shape from data.

The proper time resolution for the signal events can be described by a sum of two Gaussian functions ${ }^{3}$ with the resolutions of $52 \pm 5$ and $114 \pm 7 \mathrm{fs}$, and the fraction of narrow component of $51 \pm 9 \%$, see Figure 1 .

\subsection{The background}

Possible backgrounds have been studied in detail both for $\mathrm{B}_{\mathrm{s}}^{0} \rightarrow \phi \gamma$ and $\mathrm{B}^{0} \rightarrow \mathrm{K}^{* 0} \gamma$ modes using slightly released selection criteria to increase the event statistics [15]. In the available $b \bar{b}$

\footnotetext{
${ }^{3}$ The $\mathrm{B}_{\mathrm{s}}^{0}$ proper time resolution is dominated by the $\phi$-vertex resolution. The vertex is reconstructed with two kaon tracks and the error on its position depends strongly on the opening angle between two kaons. In particular it increases with the decreasing of the angle between the $\phi$ and the $\mathrm{B}_{\mathrm{s}}^{0}$ flight direction in the $\mathrm{B}_{\mathrm{s}}^{0}$ rest frame $[14,16]$.
} 


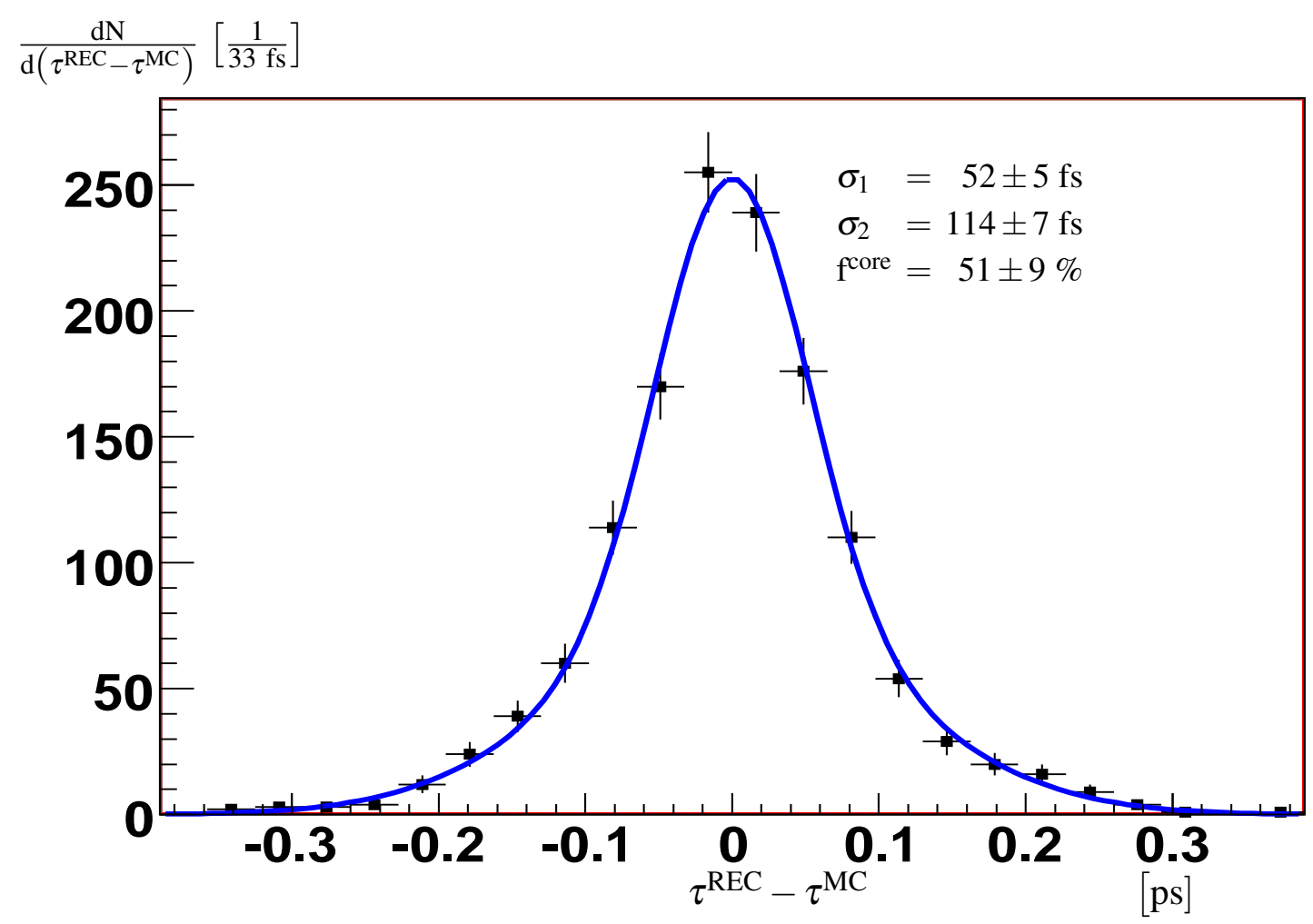

Figure 1: The proper time resolution for the $\mathrm{B}_{\mathrm{s}}^{0} \rightarrow \phi \gamma$ events passing all selection criteria [16]. The curve represents the fit with a double Gaussian function. The effective resolutions for Gaussian function are $52 \pm 5$ and $114 \pm 7$ fs and the fraction of the narrow component is $51 \pm 9 \%$.

MC the major part of the background consists of the random combination of two tracks from the same secondary vertex together with a merged $\pi^{0}$-meson, misidentified as single high energy photon. Using a dedicated $\pi^{0} / \gamma$-separation algorithm, this major fraction of background can be significantly reduced.

Peaking background from badly reconstructed $\mathrm{B}_{\mathrm{s}}^{0} \rightarrow \phi \pi^{0}$ decays where the energetic "merged" $\pi^{0}$ is misreconstructed as a photon, is expected to be small, since this decay is highly suppressed ${ }^{4}$. Even without $\gamma / \pi^{0}$-separation the cut on the polarization of the vector meson drastically reduces this potential feeddown [14]. Assuming $\mathscr{B}\left(\mathrm{B}_{\mathrm{s}}^{0} \rightarrow \phi \pi^{0}\right)=\mathscr{B}\left(\mathrm{B}^{0} \rightarrow \mathrm{K}^{* 0} \pi^{0}\right)$, and using the recent upper limit $\mathscr{B}\left(\mathrm{B}^{0} \rightarrow \mathrm{K}^{* 0} \pi^{0}\right)<3.5 \times 10^{-6}$ [22] the final contribution from this source is expected to be less than $0.4 \%$ at $90 \%$ confidence level $^{5}$.

Another background which potentially leads to a contribution in the signal mass region are misreconstructed $\mathrm{B}^{0} \rightarrow \mathrm{K}^{* 0} \gamma$ events, where the pion from the $\mathrm{K}^{* 0} \rightarrow \mathrm{K}^{+} \pi^{-}$decay is misidentified as a kaon. For a small fraction of such events the invariant mass of the true and fake kaon falls into the narrow mass window around the nominal $\phi$ meson mass. A Monte Carlo study shows that for this background the central value of the reconstructed mass is shifted by $\sim 100 \mathrm{MeV} / c^{2}$ upwards with respect to the nominal mass of the $\mathrm{B}_{\mathrm{s}}^{0}$ meson and its width is about 2-3 times wider. However, the absolute contribution is totally negligible and amounts to $0.3 \%$ even in the case of a

\footnotetext{
${ }^{4}$ This isospin-violating decay proceeds though gluonic penguin graph, which in turn preserves isospin.

${ }^{5}$ This contribution has been estimated to be less than $4 \%$ in reference [14] using $\mathscr{B}\left(\mathrm{B}^{0} \rightarrow \mathrm{K}^{* 0} \pi^{0}\right)<3.6 \times 10^{-5}$.
} 

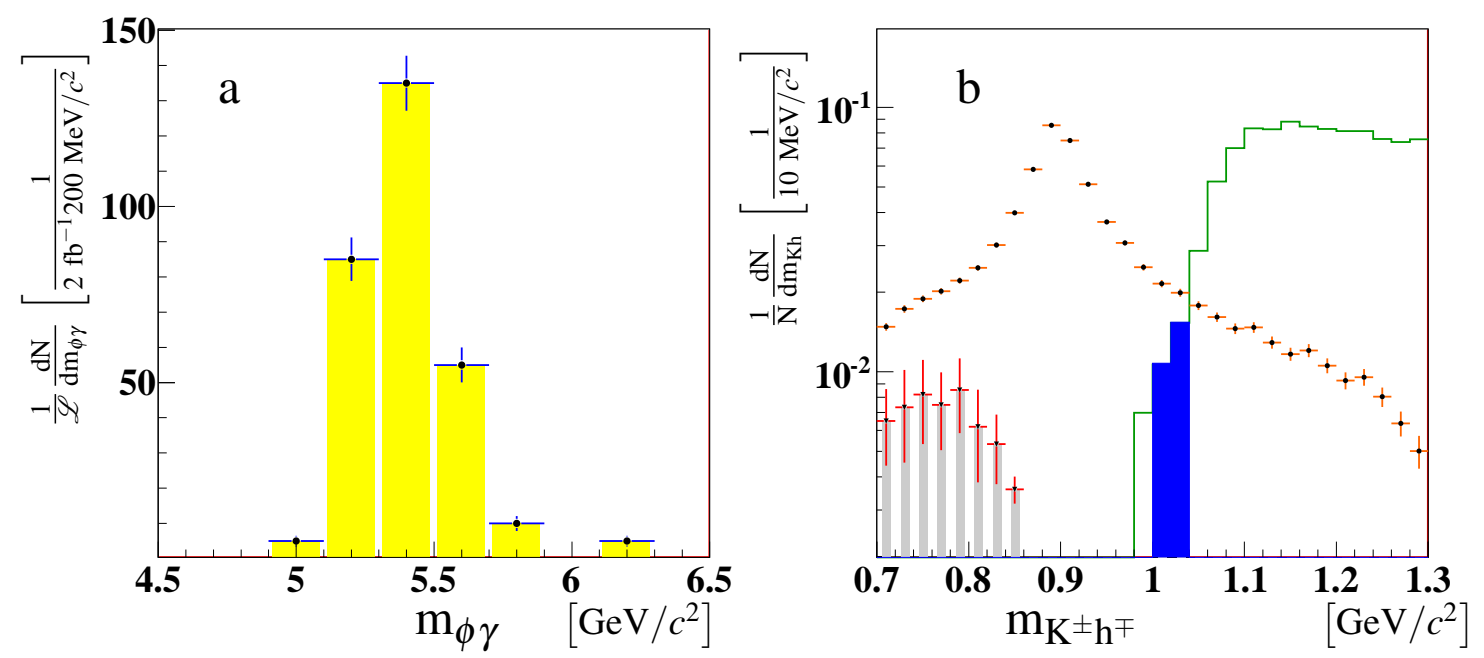

Figure 2: a) The absolute contribution of the feeddown from $\mathrm{B}^{0} \rightarrow \mathrm{K}^{* 0} \gamma$ decays misreconstructed as $\mathrm{B}_{\mathrm{s}}^{0} \rightarrow \phi \gamma$ signal events for $2 \mathrm{fb}^{-1}$. b) The distribution of the $\mathrm{K} \pi$ invariant mass from $\mathrm{B}^{0} \rightarrow \mathrm{K}^{* 0} \gamma$ decays (points with errors) and the corresponding mass distribution in case the pion is identified as second kaon (open histogram). The solid blue histogram shows the fraction of these misidentified $\mathrm{K}^{+} \mathrm{K}^{-}$combinations, consistent with the mass window used for selecting $\phi$ candidates. The light grey histogram with error bars shows the original $\mathrm{K} \pi$ invariant mass of the wrongly identified $\phi$ events. A $100 \%$ misidentification of pions as kaons has been assumed for these plots.

100\% misidentification of pions as kaons, see Figure 2.

\subsection{The statistical uncertainty in CP-asymmetry}

The studies of the photon polarisation and $\mathrm{CP}$-violation parameters in the decay $\mathrm{B}_{\mathrm{s}}^{0} \rightarrow \phi \gamma$ are described in $[14,15,16,17]$. A fast Monte Carlo simulation was performed to estimate the statistical error for the measurement of the $C, S, \mathscr{A}^{\Delta}$, using as input, the signal yield, background to signal ratio, proper time acceptance function and resolution from full simulation. Using $10^{4}$ toy Monte Carlo experiments, the statistical uncertainty in the parameters is $\mathscr{A}^{\Delta}=0.217 \pm 0.002$, $C=0.115 \pm 0.001$ and $S=0.114 \pm 0.001$ for $2 \mathrm{fb}^{-1}$.

\subsection{Conclusions}

The basic steps toward the first measurements of the radiative decays have been presented. We have concentrated on the probing of photon polarization in $\mathrm{B}_{\mathrm{s}}^{0} \rightarrow \phi \gamma$ decay. Based on our Monte Carlo simulation, for $2 \mathrm{fb}^{-1}$ we expect to reach the uncertainty in the measurement of the parameter $\mathscr{A}^{\Delta}, \sigma_{\mathscr{A} \Delta} \approx 0.22$. The largest systematic comes from the knowledge of the proper time acceptance function and is currently under study. The capability of LHCb experiment to measure this and other parameters has been discussed.

Similar studies have shown that the measurement of the direct $C P$-asymmetry for $\mathrm{B}^{0} \rightarrow \mathrm{K}^{* 0} \gamma$ decay can be performed with statistical precision around 0.018 already with a data-set of an integrated luminosity around $100 \mathrm{pb}^{-1}$, and the the measurements of ratio of branching fractions for $\mathrm{B}_{\mathrm{s}}^{0} \rightarrow \phi \gamma$ and $\mathrm{B}^{0} \rightarrow \mathrm{K}^{* 0} \gamma$ can be done with a statistical precision less than $5 \%$. 


\section{References}

[1] M. Gronau, Y. Grossman, D. Pirjol and A. Ryd, "Measuring the Photon Helicity in Radiative B Decays", Phys. Rev. Lett. 88, 051802 (2002)

[2] M. Gronau and D. Pirjol, "Photon polarization in radiative B decays", Phys. Rev. D 66, 054008 (2002)

[3] V.D. Orlovsky and V.I. Shevchenko, "On the photon polarization in radiative B $\rightarrow \phi K \gamma$ decay", Phys. Rev. D 77, 093003 (2008)

[4] T. Mannel and S. Recksiegel, "Flavour Changing Neutral Current Decays of Heavy Baryons: The Case $\Lambda_{b} \rightarrow \Lambda \gamma^{\prime}$, J. Phys. G 24, 979 (1998)

[5] G. Hiller and A. Kagan, "Probing for New Physics in Polarized $\Lambda_{b}$ decays at the Z", Phys. Rev. D $\mathbf{6 5}, 074038$ (2002)

[6] F. Legger and T. Schietinger, "Photon helicity in $\Lambda_{\mathrm{b}} \rightarrow \mathrm{pK} \gamma$ decays" Phys.Lett.B 645, 204 (2007)

[7] D. Melikhov, N. Nikitin and S. Simula, "Probing right-handed currents in $B \rightarrow \mathrm{K}^{*} \ell^{+} \ell^{-}$transitions", Phys. Lett. B 442, 381 (1998)

[8] C.S. Kim, Y.G. Kim, C.D. Lü and T. Morozumi, "Azimuthal angle distribution in $\mathrm{B} \rightarrow \mathrm{K}^{*}(\rightarrow \mathrm{K} \pi) \ell^{+} \ell^{-}$at low invarinat $\mathrm{m}\left(\ell^{+} \ell^{-}\right)$region”, Phys. Rev D 62, 034013 (2000)

[9] Y. Grossman and D. Pirjol, "Extracting and using photon polarization information in radiative B decays", JHEP 0006, 029 (2000)

[10] F. Krüger, L.M. Sehgal, N. Sinha and R. Sinha, "Angular distribution and CP asymmetries in the decays $\overline{\mathrm{B}} \rightarrow \mathrm{K}^{-} \pi^{+} \mathrm{e}^{-} \mathrm{e}^{+}$and $\overline{\mathrm{B}} \rightarrow \pi^{-} \pi^{+} \mathrm{e}^{-} \mathrm{e}^{+}$, Phys. Rev. D 61, 114028 (2000)

[11] J. Lefrancois and M.-H. Schune, "Measuring the photon polarization in $\mathrm{b} \rightarrow \mathrm{s} \gamma \mathrm{using} \mathrm{B} \rightarrow \mathrm{K}^{*} \mathrm{e}^{+} \mathrm{e}^{-}$ decay channel”, CERN-LHCb-PUB-2009-008

[12] D. Atwood, T. Gershon, M. Hazumi and A. Soni, "Mixing-induced CP violation in $B \rightarrow \mathrm{P}_{1} \mathrm{P}_{2} \gamma$ in search of clean new physics signals", Phys. Rev. D 71 (2005) 076003

[13] F. Muheim, Y. Xie and R. Zwicky, "Exploiting the width difference in $\mathrm{B}_{\mathrm{s}} \rightarrow \phi \gamma$ ”, Phys. Lett. B 664, 174 (2008)

[14] P. Pakhlova and I. Belyaev, "Radiative B decays at LHCb”, CERN-LHCb-2003-090

[15] L. Shchutska, A. Golutvin and I. Belyaev, "Study of radiative penguin decays B $\rightarrow \mathrm{K}^{* 0} \gamma$ and $\mathrm{B}_{\mathrm{s}} \rightarrow \phi \gamma$ at LHCb", CERN-LHCb-2007-030

[16] L. Shchutska et al., "Probing the photon polarization in $\mathrm{B}_{\mathrm{s}} \rightarrow \phi \gamma$ at LHCb", CERN-LHCb-2007-147

[17] L. Shchutska, "Probing the photon polarization in $\mathrm{B}_{\mathrm{s}} \rightarrow \phi \gamma$ at LHCb", Master Theses, MIPT\&ITEP/Moscow, Russia, 2008, CERN-THESIS-2008-095

[18] F. Legger, "Reconstruction of the decays $\Lambda_{b} \rightarrow \Lambda^{0} \gamma$ and $\Lambda_{b} \rightarrow \Lambda(1670) \gamma$ at LHCb", CERN-LHCb-2006-012

[19] F. Legger, "Polarized radiative $\Lambda_{b}$ decays at LHCb", CERN-LHCb-2006-013

[20] F. Legger, "Contribution to the Development of the LHCb acquisition electronics and Study of polarized radiative $\Lambda_{\mathrm{b}}$ decays", Ph.D. Theses, EPFL/Lausanna, Switzerland, 2006, CERN-THESIS-2006-044

[21] S. Amato et al., "LHCb calorimeters: Technical Design Report”, CERN-LHCC-2000-036

[22] C. Amsler et al., "Review of Particle Physics," Phys. Lett. B 667, 1 (2008) and 2009 partial update for the 2010 edition. 